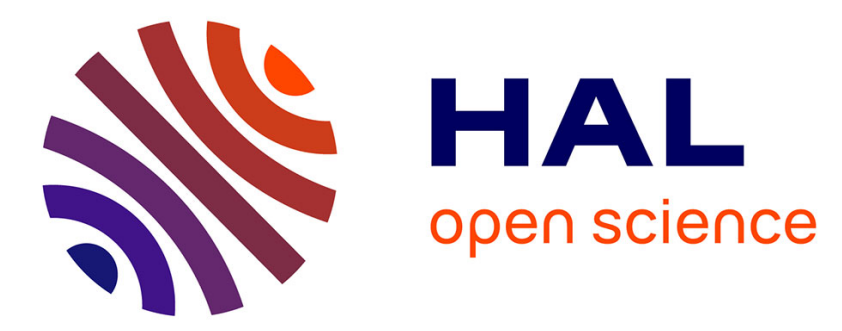

\title{
La frontière albano-grecque, histoire d'un avant-poste italien (1878-1947)
}

Fabrice Jesné

\section{To cite this version:}

Fabrice Jesné. La frontière albano-grecque, histoire d'un avant-poste italien (1878-1947). Michel Catala; Dominique Le Page; Jean-Claude Meuret. Frontières oubliées, frontières retrouvées. Marches et limites anciennes en France et en Europe, Enquêtes \& Documents (41), Presses universitaires de Rennes, pp.269-276, 2012, 978-2-7535-1739-4. 10.4000/books.pur.34319 . halshs-02288677

\section{HAL Id: halshs-02288677 https://shs.hal.science/halshs-02288677}

Submitted on 15 Sep 2019

HAL is a multi-disciplinary open access archive for the deposit and dissemination of scientific research documents, whether they are published or not. The documents may come from teaching and research institutions in France or abroad, or from public or private research centers.
L'archive ouverte pluridisciplinaire HAL, est destinée au dépôt et à la diffusion de documents scientifiques de niveau recherche, publiés ou non, émanant des établissements d'enseignement et de recherche français ou étrangers, des laboratoires publics ou privés. 
La frontière albano-grecque, histoire d'un avant-poste italien (1878-1947)

Fabrice Jesné

L'unification italienne, entre 1859 et 1870, marque l'émergence d'une nouvelle grande puissance au centre de la Méditerranée. Dès 1860, un ministre italien observait que la nouvelle configuration péninsulaire déplaçait les frontières du royaume de Savoie quasiment jusqu'à l'empire Ottoman : « La nouvelle organisation de l'Italie nous rapproche trop des frontières turques ${ }^{1}$, déclarait Giacomo Durando. La région des Pouilles, à l'extrême sud-est de la botte italienne, est en effet séparée de l'Albanie, qui se trouvait alors sous domination ottomane, par un détroit large d'une centaine de kilomètres seulement. Jusque dans le dernier quart du XIX ${ }^{\mathrm{e}}$ siècle, l'Albanie est une région extrêmement mal connue de l'Europe du Sud-Est, qui n'est recouverte par aucune délimitation administrative faisant explicitement référence aux Albanais ${ }^{2}$. La présence de populations albanaises dans les territoires ottomans proches de l'Italie fut longtemps une donnée très floue pour les Occidentaux, qui ne disposaient pour la confirmer que de lointains souvenirs historiques - ceux de Venise notamment - et de récits de voyage plus ou moins fiables ${ }^{3}$. Quoique voisins des Albanais, les Italiens s'en remirent longtemps aux savoirs produits par les autres Européens de l'Ouest; ce n'est que dans le dernier quart du XIX ${ }^{\mathrm{e}}$ siècle que l'Italie unifiée commença à élaborer un corpus de textes sur les Balkans propres à définir des intérêts nationaux dans la région qui allassent au-delà des slogans sur la lutte commune des peuples italien et balkaniques contre les empires oppresseurs $^{4}$.

L'invention de la frontière albanaise comme enjeu stratégique pour l'Italie unifiée

Dès les années 1870, l’Italie à peine née est confrontée à une crise internationale majeure, qui éclate dans une périphérie proche dont elle ne sait à peu près rien. Les provinces turques de Bosnie et d'Herzégovine s'insurgent en 1875, précipitant l'ensemble des Balkans dans une tourmente qui culmine avec la guerre russo-turque de $1877-1878^{5}$. Le congrès de Berlin qui se tient à l'été 1878 sous l'égide de Bismarck permet à l'allié austrohongrois de l'Allemagne d'occuper la Bosnie-Herzégovine, laquelle demeure formellement ottomane. Or l'empire des Habsbourgs nourrit un très profond ressentiment à l'égard de l'Italie. C'est en effet à ses dépens que s'est faite l'Unité italienne, et l'empire abrite toujours des minorités italophones. Sur la rive orientale de

1 I Documenti Diplomatici Italiani (désormais DDI) $1^{\text {re }}$ série, v. 1, d. 91, Durando à Cavour, Constantinople, 8 mai 1861 .

$2 \quad$ Parmi les provinces ottomanes, quatre étaient réputées abriter d'importantes populations albanaises : il s'agissait des vilayets de Shkodra, Kosovo, Manastir et Ioannina.

3 Notamment celui du consul Pouqueville : PouQueville F.C.H.L., Voyage en Morée, à Constantinople, en Albanie, et dans plusieurs autres parties de l'empire Othoman, pendant les années 1798, 1799, 1800 et 1801, etc., Paris, Marchant, 1805, 3 v.

$4 \quad$ L'un des tous premiers ouvrages italiens proprement consacrés à l'Albanie était dû à un patriote italoalbanais, Pietro Chiara, qui fut plus tard député et secrétaire particulier de Francesco Crispi. CHIARA P., L'Albania, Palerme, Tipografia del Giornale di Sicilia, 1869. Comme pour la quasi-totalité de la génération du Risorgimento, la Grèce représentait le phare de la liberté en Europe du Sud-Est. Cette grille de lecture conduisait Chiara à considérer la région d'Épire, aujourd'hui partagée entre l'Albanie et la Grèce, comme éminemment grecque.

TURAN Ö., (dir.), The Ottoman-Russian war of 1877-78, Ankara, Middle East University, 2007. 
l'Adriatique, le littoral austro-hongrois fait face à celui de l'Italie sur plusieurs centaines de kilomètres, et ce jusqu'aux Bouches de Kotor. Le face-à-face est toutefois très inégal, puisque la côte italienne est très basse alors que la Dalmatie comprend îles et baies par dizaines. En cas de guerre, la flotte autrichienne, quoiqu'inférieure numériquement à celle de l'Italie, pourrait donc faire irruption n'importe où et couper notamment les communications nord-sud en bombardant les voies ferrées côtières ${ }^{6}$. Cet avantage devient écrasant lorsque l'Autriche-Hongrie occupe la Bosnie et l'Herzégovine. Avec une telle profondeur stratégique, la menace autrichienne sur les côtes italiennes de l'Adriatique est en effet considérablement accrue. Pour conjurer ce cauchemar stratégique, Rome prend en 1879 le chemin d'une alliance avec Berlin et Vienne. C'est dans le cadre de cette alliance que l'Italie va s'attacher à sauver ce qui peut l'être en Adriatique, en défendant l'intégrité des provinces turques du littoral adriatique, qui correspondent pour l'essentiel à ce que nous connaissons aujourd'hui comme l'Albanie.

La crise de 1875-1878 révèle en outre l'affirmation d'un autre concurrent dans cette région, la Grèce. Dans le plus méridional des vilayets albanais, celui de Ioannina, une bonne partie de la population est en effet de langue grecque et/ou de religion orthodoxe, et comme telle revendiquée par Athènes ${ }^{7}$. C'est donc à l'occasion de cette crise que la diplomatie italienne se rendit compte de l'existence d'un double danger stratégique menaçant la sécurité de la sortie de l'Adriatique. En effet, tant l'Autriche-Hongrie que la Grèce pouvaient être, à la faveur d'une recomposition territoriale, en mesure de s'emparer de l'autre rive du canal d'Otrante et ainsi de contrôler les communications entre Adriatique et Méditerranée ${ }^{8}$. De la fin des années 1870 à la Seconde Guerre mondiale, l'Italie n'aura donc de cesse de tenter de s'assurer soit la neutralité, soit le contrôle direct ou indirect de l'autre rive du canal d'Otrante. Pour cela, Rome va s'attacher à défendre la frontière sud-occidentale de l'empire ottoman puis de l'État albanais qui lui succède.

Lors de la conférence de Berlin, la délégation italienne, emmenée par un conservateur, le comte Corti, s'était désintéressée de l'Albanie, s'attirant les foudres de la gauche italienne, dont la partie la plus modérée était au pouvoir depuis deux ans seulement ${ }^{9}$. Tout au plus Corti avait-il appuyé - avant tout par philhellénisme - la proposition française d'encourager la Grèce et la Turquie à se mettre d'accord sur un tracé frontalier. Cette décision différée permit au cours des années qui suivirent à des politiciens plus réalistes de défendre l'Albanie contre les ambitions grecques. Il faut ici rappeler que l'Italie, constituée en vertu du principe des nationalités,

$6 \quad$ Ce n'est qu'au début du XX $\mathrm{X}^{\mathrm{e}}$ siècle que la vulnérabilité de la côte adriatique devint une préoccupation majeure ; Gooch J., Esercito, Stato e società in Italia, 1870-1915, Franco Angeli, Milan, 1994, p. 176 et sq. Cf. également Friz G., MARIANO, G., La politica navale italiana dal 1885 al 1915, Rome, Ufficio storico della Marina militare, 1982.

7 Comme dans une grande partie des Balkans, la réalité ethnographique ne peut toutefois se réduire à une opposition entre blocs nationaux clairement identifiés. Le peuplement épirote prenait la forme d'un subtile camaïeux d'identités linguistiques, religieuses et territoriales à partir duquel se distinguèrent les nationalités grecque, albanaise, valaque, sans oublier les minorités juive et rom : WINNIFRITH T.J., Badlands, Borderlands. A History of Northern Epirus/Southern Albania, Londres, Duckworth, 2002, p. 121.

8 Pour Stavro Skendi, c'est à partir du début des années 1890 que le mouvement national albanais «lâche » l'empire Ottoman et commence à revendiquer la création d'un État regroupant les populations albanaises de Turquie: SKEndi S., The Albanian National Awakening. 1878-1912, Princeton, Princeton University Press, 1967, p. 316-318.

CARDElla SANTUCCI C., « La democrazia italiana e la politica estera, 1875-1878 », Thèse de doctorat, Università degli Studi di Milano, 1986 ; Celozzi BALdelli, P.G., L'Italia e la crisi balcanica (1876-79), Lecce, Marco Congedo Editore, 2000. 
était viscéralement philhellène et qu'une telle Realpolitik n'allait pas de $\operatorname{soi}^{10}$. L'attachement de l'opinion italienne pour la Grèce était surtout sentimental - cette dernière étant avant tout un symbole de la libération nationale - et le nationalisme grec avait d'ailleurs pris, comme ailleurs en Europe, une coloration de plus en plus exclusive et agressive ${ }^{11}$. En somme, en défendant les intérêts stratégiques de l'Italie, le gouvernement italien paraissait, aux yeux de sa propre opinion publique, bafouer les droits de la Grèce, alors qu'en réalité il défendait par la même occasion ceux du peuple albanais, qui paraissait aux yeux de la plupart des observateurs majoritaire dans la zone.

Il faut ici évoquer le rôle fondamental que jouèrent les consuls italiens dans l'invention de la frontière albanogrecque. L'Italie unifiée installa assez rapidement des consuls dans les chefs-lieux des vilayets albanais occidentaux : à Shkodra en 1861-62, à Ioannina en 1870. Il fallut en revanche attendre la toute fin du siècle pour que l'Italie installe des représentants dans l'intérieur albano-macédonien, à Monastir en 1895, puis en 1902 à Usküb. Le réseau était complété par des délégations consulaires à la localisation fluctuante, qui concerna essentiellement les localités côtières de Durrës, Lezha, Préveza et Vlora ${ }^{12}$. Les consuls furent les principaux agents de la défense des intérêts italiens en Albanie, et les artisans de l'édification d'une frontière la plus sûre possible pour l'Albanie. Évoquons à titre d'exemple le rôle joué par le consul italien Enrico De Gubernatis dans le tracé de la frontière turco-grecque de $1881^{13}$. Ethnologue amateur, il réalisa une «Carte ethnographique de l'Épire» opportunément publiée en 1879 alors qu'une commission européenne avait été mandatée par la conférence de Berlin pour tracer la frontière gréco-turque de façon à respecter le principe des nationalités. En l'absence de véritables connaissances géographiques et ethnographiques sur la région, les puissances européennes partaient du principe que l'Épire était une région essentiellement hellénique qui devait revenir à la Grèce. Un tel jugement reposait avant tout sur les souvenirs de l'antiquité, mais aussi sur la croyance, alors assez fréquente, que les Albanais étaient apparentés aux Grecs ${ }^{14}$. En tant qu'expert de la zone, De Gubernatis fut rappelé en Épire par le président du Conseil italien, Agostino Depretis, qui entendait se faire une idée exacte de la situation sur le terrain. De Gubernatis conclut à l'imbrication étroite des diverses nationalités: Albanais, Grecs, mais aussi Valaques. Les populations avaient surtout des identités locales, souvent mixtes, étrangères aux catégories que les comités panhelléniques venus de Grèce voulaient leur faire adopter. Seul le chef-lieu de

10 Collectif, Indipendenza e unità nazionale in Italia ed in Grecia. Convegno di studio. Atene, 2-7 ottobre 1985, Florence, L. S. Olschki, 1987 ; GuIDA F., « Italia e Grecia dalla formazione del Regno di Grecia ai giorni nostri », in « Le relazioni tra l'Italia e la Grecia », n ${ }^{\circ}$ spécial de Il Veltro, 27-1/2, 1983, p. 27-55; LiAKOS A., L'unificazione italiana e la Grande Idea. Ideologia e azione dei movimenti nazionali in Italia e in Grecia, 1859-1871, Florence, Aletheia, 1995 ; LuCARELli E., SPETSIERI C., (dir.), Risorgimento greco e filellenismo italiano. Lotte, cultura, arte. mostra promossa dall'Ambasciata di Grecia e dall'Associazione per lo sviluppo delle relazioni fra Italia e Grecia. Roma, Palazzo Venezia, 25 marzo - 25 aprile 1986, Rome, Edizioni del Sole, 1986; PeCOUT G., «Une amitié politique méditerranéenne: le philhellénisme italien et français au XIX ${ }^{\mathrm{e}}$ siècle », «Annali » della fondazione Giangiacomo Feltrinelli, 2003, p. 81-106, p. 84.

11 Sigalas N., «Hellénistes, hellénisme et idéologie nationale. De la formation du concept d'« hellénisme » en grec moderne », in AVLAMI Ch., (dir.), L'Antiquité grecque au XIX siècle. Un exemplum contesté ?, Paris, L'Harmattan, 2000, p. 239-291.

12 Pour une présentation détaillée de la politique consulaire et diplomatique de l'Italie libérale dans l'espace albanais, nous nous permettons de renvoyer à notre thèse : JESNE F., «Les nationalités balkaniques dans le débat politique italien, de l'Unité au lendemain des Guerres Balkaniques (1861-1913) », Université de Paris 1, 2009.

$13 \quad I b .$, p. 878

14 Cf. par exemple les travaux d'un anthropologue napolitain sur la question: NicolucCI G., Sull'antropologia della Grecia, Naples, Stamperia del Fibreno, 1867. 
Ioannina paraissait pleinement grec : dans le sud des Balkans, urbanité, modernité et hellénisme allaient en effet le plus souvent de pair. De telles conclusion allaient pleinement dans le sens des intérêts stratégiques italiens au sortir de l'Adriatique, et le gouvernement de Rome s'en servit pour affronter la Grèce, les grandes puissances philhellènes et sa propre opinion publique. La démarche fut payante, puisque l'Italie obtint, contrairement à l'hypothèse de départ de la conférence de Berlin, que la Grèce acquiert la Thessalie mais pas l'Épire ${ }^{15}$.

La frontière de 1913

Les frontières de Berlin parvinrent tant bien que mal à se maintenir jusqu'aux guerres balkaniques de 1912-1913, qui remirent tout en question. Entretemps, la situation stratégique de l'Italie avait beaucoup changé. Le pays avait connu en effet un décollage économique dans les premières années du $\mathrm{XX}^{\mathrm{e}}$ siècle, qui lui offrit en peu de temps un net surcroît de puissance, même si l'Italie demeurait la plus faible des grandes puissances ${ }^{16}$. En politique étrangère, cela se traduisit par deux phénomènes marquants : la relance de la colonisation, avec la saisie de la Tripolitaine et de la Cyrénaïque en 1911-12, et une politique plus ferme à l'égard de l'Autriche-Hongrie dans les Balkans, même si le containment restait à l'ordre du jour.

Dans la région, l'Italie dut toutefois de plus en plus compter avec la Grèce, qui était l'un des grands vainqueurs des Guerres Balkaniques. Le royaume hellénique était devenu un rival stratégique dangereux, dans la mesure où il était en position de s'emparer du détroit de Corfou. En effet, la Grèce contrôlait l'archipel ionien depuis que le Royaume-Uni le lui avait cédé en 1864. L'effondrement ottoman de 1912-1913 permit en outre à ses troupes de remonter en Épire du nord ${ }^{17}$. Si la Grèce parvenait à s'installer dans la zone, cela signifierait qu'elle pourrait y installer une base navale qui lui permettrait de menacer la sortie de l'Adriatique. Elle représenterait dès lors une menace pour l'Italie, mais aussi pour l'Autriche-Hongrie, qui, malgré ses efforts et ceux de l'Allemagne, courrait le risque que la Grèce choisisse le camp de la France et de la Russie. A l'issue de la deuxième guerre balkanique, le sort de la Turquie d'Europe fut réglé par les grandes puissances au cours d'une série de conférences. Les conquêtes des vainqueurs furent entérinées pour l'essentiel, à l'exception des territoires peuplés d'Albanais ${ }^{18}$. Sous la pression de l'Autriche-Hongrie et de l'Italie, en effet, on évita qu'ils fussent partagés entre les clients serbes et grecs de l'Entente. Vienne et Rome durent cependant lutter pied à pied pour obtenir les frontières les plus favorables possibles pour l'Albanie. Au nord, elles durent faire la part du feu et céder le Kosovo à la Serbie.

15 «Convention pour régler les questions relatives à la rectification des frontières turco-grecques, suivie d'un Acte séparé concernant l'évacuation et la prise de possession des territoires cédés à la Grèce, ainsi que d'un Protocole supplémentaire ; signée à Constantinople, le 24 mai 1881 », in MARTENS, G.Fr. de, Recueil général de traités et autres actes relatifs aux rapports de droit international, $2^{\mathrm{e}}$ série, v. 6, Nendeln - New York, Kraus Reprint Limited - Johnson Reprint Corporation (Gottingen, 1881), p. 753-760.

${ }_{16} \quad$ Sur les liens entre expansion économique et politique balkanique de l'Italie libérale, cf. la thèse contestée de Richard Webster et le bilan complet établi par Daniel Grange : WeBSTER R.A., L'imperialismo industriale italiano, studio sul prefascismo, Turin, Einaudi, 1974 ; GRANGE D.J., L'Italie et la Méditerranée, 1896-1911. Les fondements d'une politique étrangère, Rome, École française de Rome, Collections de l'École française de Rome, n¹97, 1994, «"Un posto al sole". Zones d'action et sphères d'influence en Méditerranée. Livre 1, les Balkans », p. 1201-1346.

17 Sur les inquiétudes italiennes quant à la politique grecque en Épire au moment des guerres balkaniques, cf. JESNE F., « Militaires et diplomates italiens face aux déplacements contraints de populations dans les Balkans. Enjeux politiques et territoriaux », in FORCADE O., (dir.), Les réfugiés dans l'histoire moderne et contemporaine, Paris, Nouveau Monde Éditions, 2008, p. 113-130.

$18 \quad$ BIAGINI A., L' Italia e le guerre balcaniche, Rome, USSME, 1990 ; IVETIC E., Le guerre balcaniche, Bologne, Il mulino, 2006. 
Au sud, cependant, l'Autriche-Hongrie et l'Italie eurent partiellement gain de cause. L'État-major italien était en particulier très hostile à la Grèce ${ }^{19}$. L'armée et la marine italienne réussirent donc à convaincre le gouvernement, qui était prêt à faire des concessions en Albanie en échange du soutien de l'Entente à ses revendications sur le Dodécanèse, de ne pas transiger sur la frontière méridionale de l'Albanie ${ }^{20}$. Aussi l'actuel tracé de cette frontière est-il dû à la volonté de l'Italie d'empêcher l'émergence d'un concurrent supplémentaire à sa frontière maritime orientale.

Frontière albanaise et protectorat italien

La comparaison du tracé des frontières de 1913 avec le tracé actuel donne l'image d'une permanence remarquable. Cette image ne doit pas pour autant éclipser les tentatives italiennes de modification du tracé, qui furent nombreuses et prirent deux directions apparemment contradictoires. La politique albanaise de l'Italie oscilla en effet entre la tentation d'amputer le territoire albanais au profit de l'Italie d'une part, et la volonté de défendre et d'étendre le territoire albanais face à la Grèce d'autre part. La contradiction, toutefois, n'est qu'apparente. En effet, l'attachement sincère de l'Italie libérale au principe des nationalités s'accommode de revendications impérialistes sur l'Albanie dans la mesure où la nation albanaise est inventée comme parente mineure de la nation italienne, qu'il convient de protéger et d'accompagner sur la voie de la «civilisation». Cette rhétorique sera reprise et aménagée par le fascisme, dont les pratiques seront toutefois infiniment plus brutales et déboucheront sur l'invasion et l'annexion du pays en 1939. Il convient toutefois de rappeler les modifications formelles et informelles que subit la frontière albano-grecque entre 1913 et 1947.

Jusqu'au début de la Première Guerre mondiale, la situation restait celle d'une rivalité austro-italienne pour le contrôle du faible État albanais. Le conflit mondial marqua toutefois la fin de la cogestion internationale du pays et permit à la Grèce de faire avancer, de façon plus ou moins masquée, ses troupes en Épire. Au sein de la sphère de décision italienne, de plus en plus de voix réclamèrent la constitution d'une zone d'influence en Albanie du sud autour du port de Vlora qui fait face à Otrante ${ }^{21}$. La nouvelle équipe qui, à l'automne 1914, prit la tête de la diplomatie romaine, s'orienta rapidement vers une prise de gages territoriaux en Albanie du sud, avec l'occupation de l'ilot de Sasen le 30 octobre 1914 et celle du port de Valona à la fin du mois de décembre de la

19 Cf. par exemple Archivio del Ufficio Storico dello Stato Maggiore, G33/17, cart. 5, Appunti tratti da una memoria del capo di Stato Maggiore della marina (« Notes tirées d’un mémoire du chef d'État-major de la marine »), Rome, Novembre 1917. L'« objectif principal» y était défini comme suit: «contraindre le gouvernement grec à se plier à notre volonté, au moyen d'une action éclair, vaste et à même de frapper le pays dans ses intérêts les plus vitaux ».

20 Le président du Conseil Giovanni Giolitti était prêt à sacrifier l'Albanie ; c'est le ministre des Affaires étrangères, Antonino di San Giuliano, qui fit valoir auprès de lui la nécessité d'y défendre les intérêts de l'Italie. Archivio Storico del Ministero degli Affari Esteri, Archivio di Gabinetto 1910-1920, casella 27, fasc.314; Ferraioli G., Politica e diplomazia in Italia tra XIX e XX secolo. Vita di Antonino Di San Giuliano (18521914), Soveria Mannelli, Rubbettino, 2007 ; MAZZETTI M., «L'Italia e la crisi balcanica del marzo-maggio $1913 »$, Storia contemporanea, 1973-2, p. 219-262. 21 était en revanche partisan d'un partage de l'Albanie entre les États balkaniques voisins afin de se concilier leurs bonnes grâces contre l'Autriche; dans sa correspondance avec le directeur du journal, Luigi Albertini, il se désolait de ce que le ministre des Affaires étrangères, Sidney Sonnino, ait au contraire choisi d'intervenir en Albanie: AlberTini L., Epistolario 1911-1926. V. 1, Dalla guerra di Libia alla Grande Guerra, édité par Ottavio Barié, Arnaldo Mondadori Editore, 1968, d. 255, 256, 302. 
même année $e^{22}$. Pendant de longs mois, la position des troupes italiennes demeura précaire, jusqu'à ce que Rome se rallie à l'Entente, grâce à la signature du pacte de Londres le 26 avril 1915. Par cette convention secrète, l'Italie se voyait octroyer différents territoires, dont la région de Valona ainsi qu'un protectorat sur un État albanais réduit à l'Albanie centrale, le reste étant partagé entre les voisins balkaniques ${ }^{23}$. L'évolution du conflit rendit ce projet caduc, et l'Albanie, envahie par l'Autriche-Hongrie en 1916 et défendue au sud par les troupes italiennes et françaises, recouvrit progressivement ses frontières initiales ${ }^{24}$. Admise à la SDN en 1920, l'Albanie était toujours partiellement occupée par la Yougoslavie et la Grèce. Le 9 novembre 1921, la conférence des ambassadeurs des grandes puissances reconnaissait les frontières de 1913, qui devaient être précisées par une commission d'enquête. Surtout, ce protocole stipulait que toute violation des frontières albanaises constituait une menace pour la sécurité italienne. Avec l'instauration du régime fasciste, Rome allait se prévaloir de ce texte pour considérer disposer en Albanie d'un « quasi mandat ${ }^{25}$.

L'agression mussolinienne de 1939 se solda à la fois par une disparition totale de la souveraineté albanaise, et par une extension momentanée des frontières du royaume d'Albanie dont la couronne avait été ceinte par VictorEmmanuel III. La destruction de la Yougoslavie par l'Axe permit en effet d'étendre son protectorat albanais grâce à l'annexion du Kosovo ${ }^{26}$. Un tel programme prévoyait également au départ l'annexion des territoires épirotes cédés à la Grèce en 1912-13, et qui abritaient une importante minorité albanophone et musulmane, les $\mathrm{Tchams}^{27}$. C'est d'ailleurs sous le prétexte de satisfaire les revendications des irrédentistes albanais que l'Italie avait agressé la Grèce en $1940^{28}$. La défaite de l'Italie fasciste et de l'Allemagne nazie se solda pour l'Albanie par une restauration de son indépendance et un retour aux frontières de $1938^{29}$. Néanmoins, comme partout en Europe orientale, les populations considérées comme « collaboratrices » furent durement sanctionnées par les vainqueurs. C'est ainsi que les populations tchames de Grèce, déjà victimes de déplacements et d'assimilation forcés entre les deux guerres, disparurent presque entièrement de Grèce. C'était là le dernier acte de la disparition des anciennes frontières ottomanes que l'Italie libérale puis le fascisme avaient tenté d'instrumentaliser au profits de leurs intérêts stratégiques. C'est in fine l'ancienne mixité culturelle héritée des vieux empires qui en fut la principale victime, au profit du modèle de l'État-nation monoethnique, dont la

$22 D D I, 5^{\text {e }}$ série, v. 2 , d. $313,366,416,457$ et 484 notamment.

23 PASTORELli P., L'Albania nella politica estera italiana 1914-1920, Naples, Jovene, 1970, p. 1-36.

24 AUGRIS E., "Korçë dans la Grande Guerre, Le sud-est albanais sous administration française (19161918) », Balkanologie, 4-2, décembre 2000, http://balkanologie.revues.org/index315.html ; PoPESCU S., "Les Français et la république de Kortcha (1916-1920) », Guerres mondiales et conflits contemporains, 2004-1, p. 7982 ; UfFicio StOrico Dello StATo MAGgiore Dell'Esercito, L'esercito italiano nella Grande Guerra (19151918). VII, Le operazioni fuori del territorio nazionale, Albania - Macedonia - Medio Oriente, Rome, Rome, USSME, 1981-1983, 3 v.; VILlARI, (Giovanni), «La presenza italiana in Albania 1918-1920», Italia Contemporanea, 256-257, septembre-décembre 2009, p. 524-536.

25 AnONYMe, Albania, Milan, Istituto per gli Studi di Politica Internazionale, 1940, p. 81-82.

26 Rodogno D., Il nuovo ordine mediterraneo. Le politiche di occupazione dell'Italia fascista (19401943), Turin, Bollati Boringhieri, 2002, 586 p., p. 352-360.

27 Ib., p. 137-141.

28 Le 15 octobre 1940, Mussolini réunit le ministre des Affaires étrangères Ciano, le Lieutenant général en Albanie Jacomoni ainsi que l'État-major afin de définir les modalités et les objectifs de l'attaque contre la Grèce: $D D I, 9^{\mathrm{e}}$ série, v. 5, d. 728. Quelques jours plus tard, le comte Ciano récapitulait les objectifs territoriaux : « Nous réclamons l'annexion à l'Albanie des territoires compris en-deçà d'une ligne Florina-PindeArta-Préveza ainsi que l'annexion à l'Italie des Îles Ioniennes », ib., d. 958, Ciano a Mussolini.

29 Par l'article 27 du traité de paix signé à Paris le 10 février 1947 par les anciens alliés du III ${ }^{\mathrm{e}}$ Reich, L'Italie « [reconnaissait] la souveraineté et l'indépendance de l'État albanais et [s'engageait] à les respecter». 
transposition en Europe du Sud-Est à été la source de conflits souvent perçus à tort comme essentiellement «balkaniques $»^{30}$.

30 Todorova, (M.), Imagining the Balkans, New York - Oxford, Oxford University Press, 1997, p. 117119. 